\title{
Is Going Unnoticed More Socially Acceptable?: An Exploration of the Relationship Between Social Acceptability and Noticeability of Fitness Trackers
}

\author{
Yumiko Sakamoto ${ }^{*}$ Pourang Irani ${ }^{*}$ and Khalad Hasan ${ }^{\dagger}$ \\ *University of Manitoba, Winnipeg, Manitoba, Canada \\ ${ }^{\dagger}$ University of Biritish Columbia, Okanagan, British Columbia, Canada \\ umsakamo@umanitoba.ca, pourang.irani@cs.umanitoba.ca, \\ khalad.hasan@ubc.ca
}

\begin{abstract}
While fitness trackers are becoming increasingly popular, the majority of such devices are relatively smaller and almost always worn around a user's wrist (e.g., smart watches). To expand the potential of novel design options for such devices, a study explored the link between social acceptability and device noticeability, in conjunction with two other factors; namely, the device size and the on-body location (i.e., on which body parts the user wears the tracker). The central question we investigated was: to develop a socially acceptable fitness tracker, should the device be less noticeable? For this exploration, an online questionnaire was distributed $(N=32)$, and results indicated that noticeability was correlated with social acceptability only in two situations: i) when the fitness tracker is large, or ii) when a female user wears it around their chest. That is, noticeability partially accounted for social acceptability only in these conditions. Jointly, the results point toward the great possibility for
\end{abstract}

How to cite this book chapter:

Sakamoto, Y., Irani, P. and Hasan, K. 2020. Is Going Unnoticed More Socially Acceptable?: An Exploration of the Relationship Between Social Acceptability and Noticeability of Fitness Trackers. In: Loizides, F., Winckler, M., Chatterjee, U., Abdelnour-Nocera, J. and Parmaxi, A. (eds.) Human Computer Interaction and Emerging Technologies: Adjunct Proceedings from the INTERACT 2019 Workshops. Pp. 117-124. Cardiff: Cardiff University Press. DOI: https://doi.org/10.18573/book3.n. License: CC-BY 4.0. 
novel design ideas of fitness trackers in other conditions (e.g., when the device is smaller or worn around the arm) without compromising social acceptability.

\section{Keywords}

Social Acceptability $\cdot$ Noticeability $\cdot$ Health and Fitness Tracker

\section{Introduction}

Over the last decade, technology has drastically changed the way we use personal health and fitness devices, and its associated software. Today, people use a wide range of fitness tracking devices to monitor their health condition, and/ or maintain their motivation towards improving their health-related behaviors (e.g., eating nutritious food). These devices are commonly available in different form factors to nicely fit in various contexts. For instance, people often use small fitness bands on their wrist to continuously monitor their health status [4]. Similarly, many people attach their smartphones to their upper arm with armbands while they are exercising. Although it largely depends on the device size and the on body location (i.e., on which part of the body the device is worn), these devices are only slightly visually noticeable. Presumably, this is the case as one of the key factors for product success for such devices is social acceptability (i.e., How comfortable one feels about using a technology in a given social context) especially because fitness-tracking devices are often worn in public.

Understanding factors that affect user acceptance of new technology have received extensive attention recently. For instance, Rico et al. [9] investigated design dimensions that are related to smartphone gesture acceptance in various settings and revealed that user's location and the audience around the user are key factors for individual's preferred gestures. Likewise, Ahlström et al. [1] investigated mid-air gesture sizes that are socially acceptable in assorted locations and the types of audience. They found that small gestures are more acceptable than large ones, and people are more comfortable performing any of such gestures in a private space (e.g., home), and in front of familiar faces (e.g., friends). Thus, there are numerous works focusing on the acceptance of new technology, but, with only little known about the possible link between social acceptability and noticeability of devices.

In this paper, we investigate whether there is a relationship between noticeability and social acceptability in regards to health and fitness trackers. More specifically, we investigate whether the size of the device and the location of the device on the body could impact social acceptance. To do so, we conduct a study to collect participants' feedback on noticeability and social acceptability, asking them to imagine they are wearing different sized devices on different 
body parts. Our results indicate that social acceptability and noticeability of health and fitness tracking device are negatively correlated when i) the device is larger and ii) the device is worn around the chest specifically by female users. Based on these results, we generate design guidelines and recommendations for developing health and fitness trackers without compromising the levels of social acceptability.

\section{Related Work}

\subsection{Social Acceptability}

Social acceptability, or individuals' psychological comfort level towards technology use in social contexts, has been widely explored. Researchers often examine factors affecting the levels of social acceptability regarding the users' experiences of using new input devices. They have studied social acceptance and factors that influence users' willingness to use such input methods for interacting with devices. For instance, researchers investigated users' acceptance of device and body-centric gestures (e.g., tap on the nose) [9], around-device mid-air gestures [1-3] for interacting with smartphones [1] and smartglasses [2-3]. Their exploration primarily concentrated on gesture properties, such as gesture size and gesture location, that are socially acceptable in a wide range of usage contexts. Additionally, they explored how acceptability changes across user groups (e.g., family, friends, strangers), locations (e.g., private vs. public space) and users' perspective (performer vs. observer). Their results revealed that gesture properties, user groups, and location affect users' attitude towards using the input method/device. Our study is inspired by these research, and we investigated the relationship between users' social acceptability and the device noticeability.

\subsection{Noticeability}

As smart devices come in different shapes and sizes, a few recent studies examined noticeability of the devices themselves and the interaction methods with the devices. Researchers have often suggested that mobile devices and the interaction methods with the device should be unnoticeable. For instance, researchers suggested the devices [8], as well as the interaction methods [6, 10 ], need to be as natural, unobtrusive, and unnoticeable as possible to be used comfortably by users in diverse social contexts. Furthermore, users' preference for wearing devices on different on-body locations could influence the noticeability levels [9-10]. To further understand the social acceptability-noticeability relationship, in this paper, we aim to explore how varying the device size, and on-body locations affect this link. 


\section{Study}

An online-based questionnaire was distributed to explore the relationship between social acceptability and device noticeability by manipulating participants' perceived device size and on-body location. The questionnaire was divided into three major sections. The first section asked for participants' demographic information. The second and third sections assessed the social acceptability and noticeability levels for different sized fitness devices and on body locations, respectively.

\subsection{Participants}

Participants were recruited from a local university $(N=32)$ with an equal maleto-female ratio. Their age ranged between 23 and $48(M=30.69 ; S D=6.94)$. Approximately $44 \%$ of the participants had no prior experience in using fitness tracking devices, and about $31 \%$ of the participants used such devices for approximately one year.

\subsection{Correlation between social acceptability \& noticeability when the device size varies}

Size-specific social acceptability of the fitness tracker was assessed with a question: "Now, please select an appropriate number to indicate "How socially comfortable you would feel using a fitness tracking device that is in size.' That is, how comfortable do you feel about wearing these devices in public?" Three sizes were selected for our exploration (Small, Medium, and Large). To somewhat homogenize participants' perception on device size, three sample images were provided along with the scale (See Fig. 1a). Participants used a 7-point Likert scale where 1 was "Very Uncomfortable" and 7 was "Very Comfortable."

For the size-specific noticeability, the participants were asked to respond to a question; "Please imagine how noticeable the device would be if it was worn
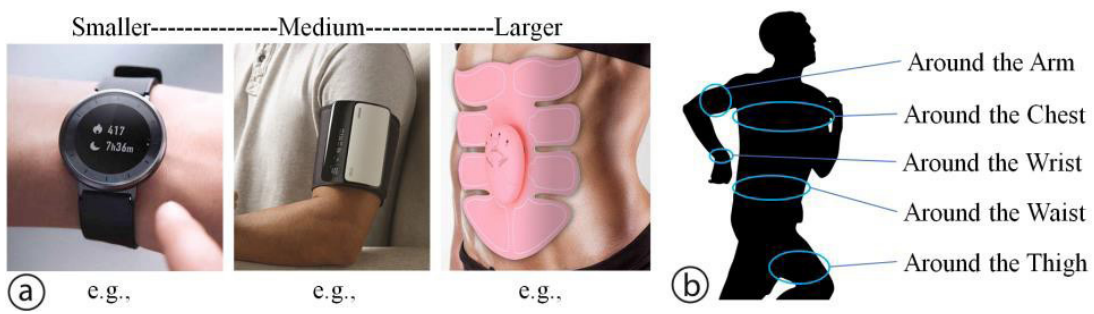

Fig. 1: (a) The scale with sample images to standardize the participants' size perception and (b) On body location image provided to the participants. 

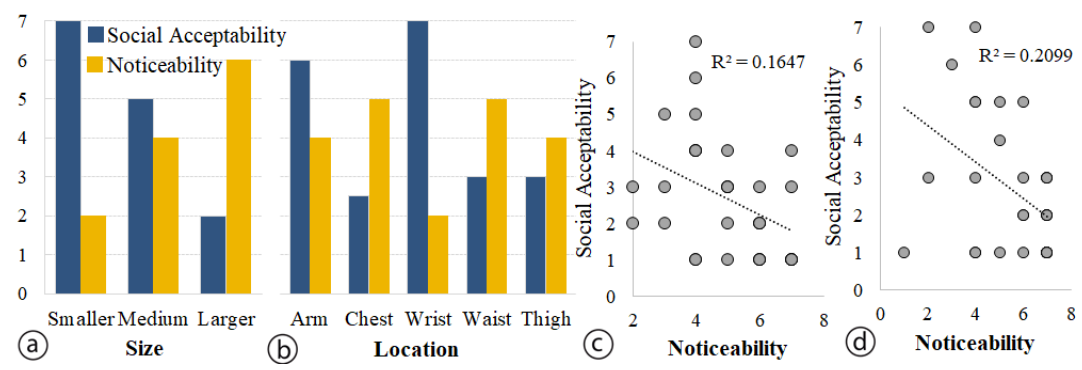

Fig. 2: Medians for social acceptability and noticeability for three device sizes (a), and five on body locations (b); Significant correlations found between social acceptability and noticeability when the device was larger (c) and the device was worn around the chest (d).

in a gym for each of the three sizes specified above. Participants used a 7-point Likert scale where 1 was "Very Unnoticeable" and 7 was "Very Noticeable." Since Kolmogorov Smirnov tests indicated the entire data were not normally distributed $(p s<.05)$, nonparametric analyses were conducted throughout the study.

Spearman's rank-order correlations explored the relationship between social acceptability and noticeability. The level of social acceptability was negatively correlated with noticeability only when the device was larger $(r s=-.43, N=$ $32, p<.01)$. Thus, only when the size of the device was larger, highly noticeable devices were perceived as not socially acceptable. In contrast, when the devices were smaller or medium-sized, highly noticeable device was not necessarily correlated with low social acceptability.

\subsection{Link between on body location \& social acceptability}

Here, we explored the link between social acceptability and noticeability based on body locations. For this, participants read the following preamble prior to answering the questions in the next section: "Please imagine that these tracking devices are visible to the people around you in a gym while you are using them." Subsequently, participants were asked to "Select an appropriate number to indicate how socially comfortable you would feel using a fitness tracking device that is attached to your." Below this question, participants saw Fig. 1(b). They used a Likert scale where 1 was "Very Uncomfortable" and 7 was "Very Comfortable" based on the body parts they imagined to wear the device (i.e., Around the; Arm, Chest, Wrist, Waist, and Thigh).

Spearman's rank-order correlations were conducted. The level of social acceptability was negatively correlated with noticeability only for the chest location $(r s=-.65, N=32, p<.01)$. That is, only for the chest-worn devices, more noticeable devices are perceived as less socially acceptable. For other locations, noticeability and social acceptability were not linked $(p s>.05)$. 
Remarkably, this finding was consistent with some of the open-ended responses where participants responded to the following question: "Please tell us your ideas about: What makes certain wearable devices socially uncomfortable to wear, even when they function very well?" (E.g., "if they are closer to more sexualized body parts, especially for females" "around chest"). Accordingly, we re-ran a Spearman's rank-order correlation while splitting the data by gender. For female, social acceptability and noticeability were strongly correlated ( $r s=-.65 ; n=16, p=.007)$ while there was no such correlation for male counterparts; $(r s=-.18 ; n=16, p=.50)$.

\section{Discussion and Design Guidelines}

This study explored the relationship between social acceptability and noticeability. Although, intuitively, noticeability might give the impression to be negatively correlated with social acceptability, our findings suggested that social acceptability and noticeability of fitness tracking devices are negatively correlated only when the devices are i) larger, or ii) worn around the chest specifically by female users.

When the devices are smaller to medium size, however, the expected correlation did not emerge. This potentially infers that when the devices are smaller to medium size, participants might not perceive the noticeable devices as socially unacceptable. Furthermore, the gender effect we discovered for the devices around the chest points toward a potential design solution: Chest worn fitness tracking devices could be designed differently for male and female users to improve the level of social acceptability. Specifically, for female users. Around the chest, devices should be particularly inconspicuous to be worn by them, while the device noticeability might not influence the male users' social comfort level as much.

In sum, our findings offer the following guidelines to designers and researchers of health and fitness tracking devices:

Device Size: use the smallest size as possible, since the acceptability decreases significantly with increased device size.

On Body Location: wearing fitness tracking devices on the upper arm or the wrist is more socially acceptable than other body parts such as the chest. Additionally, devices worn around the chest should be particularly less noticeable for female users.

Gender: researchers should pay closer attention to gender concerns while conducting social acceptability research as the acceptability ratings might vary across genders.

\section{Limitation and Future Work}

While the sample size $(N=32)$ used in our study was somewhat consistent with comparable HCI studies [5], we acknowledge that having a larger sample would 
further reinforce our claims, especially for non-significant results. Next, since all the participants were living within a Western culture, we would be wary of generalizing the study results across different cultural contexts. While exploring culturally motivated perceptual differences in acceptability and noticeability would be challenging, it would be a very fruitful path for future work. Finally, we acknowledge that this study was a computer-based questionnaire, and thus, a future laboratory experiment where participants actually experience using the fitness tracker is crucial to draw final conclusions.

\section{Conclusions}

Fitness tracking devices are evolving rapidly and becoming popular gradually, largely due to technological advances. However, such devices are almost always small and worn around the wrist. Oher potential on body locations remain mainly unexplored, presumably due to perceived lower social acceptability associated with other on body locations and device size. Indeed, it is intuitive to feel that highly noticeable devices are socially unacceptable. However, such negative correlations were not found for smaller to medium-sized devices, or around the arm, wrist, waist, and thigh devices. In sum, our results point toward great design potential for fitness tracking devices: even when the devices are noticeable, they may be perceived as socially acceptable, possibly because of other factors underlying the design of the device, when they are smaller or medium, and/ or when they are worn on the arm, wrist, waist or thigh. Future studies need to explore the contributing factors for noticeability (e.g., color and shape).

\section{Acknowledgment}

We thank all the participants for their time and feedback. This research was partially funded by a Natural Sciences and Engineering Research Council (NSERC) grant.

\section{References}

1. Ahlström, D., Hasan, K., and Irani, P.: Are you comfortable doing that?: acceptance studies of around-device gestures in and for public settings. In Proceedings of the 16th international conference on Human-computer interaction with mobile devices \& services (MobileHCI '14). pp. 193-202. ACM.

2. Alallah, F., Neshati, A., Sakamoto, Y., Hasan, K., Lank, E., Bunt, A., and Irani, P.: Performer vs. observer: whose comfort level should we consider when examining the social acceptability of input modalities for head-worn display?. In Proceedings of the 24th ACM Symposium on Virtual Reality Software and Technology (VRST '18), 9 pages. ACM. 
3. Alallah, F., Neshati, A., Sheibani, N., Sakamoto, Y., Bunt, A., Irani, P. and Hasan, K.: Crowdsourcing vs Laboratory-Style Social Acceptability Studies?: Examining the Social Acceptability of Spatial User Interactions for Head-Worn Displays. In Proceedings of the 2018 CHI Conference on Human Factors in Computing Systems (CHI '18). Paper 310, 7 pages. ACM.

4. Amini, F., Hasan, K., Bunt, A., and Irani, P.: Data Representations for In-Situ Exploration of Health and Fitness Data. In Proceedings of the 11th EAI International Conference on Pervasive Computing Technologies for Healthcare (PervasiveHealth 2017). pp. 163-172 ACM.

5. Caine, K.: Local Standards for Sample Size. In Proceedings of the SIGCHI Conference on Human Factors in Computing Systems (CHI '16). pp. 981-992. ACM.

6. Costanza, E., Inverso, S., and Allen, R.: Toward subtle intimate interfaces for mobile devices using an EMG controller. In Proceedings of the SIGCHI Conference on Human Factors in Computing Systems (CHI '05). pp. 481-489. ACM.

7. Koelle, M., Heuten, W., and Boll, S.: Are you hiding it?: usage habits of lifelogging camera wearers. In Proceedings of the 19th International Conference on Human-Computer Interaction with Mobile Devices and Services (MobileHCI '17). Article 80, 8 pages. ACM.

8. Rekimoto, J.: GestureWrist and GesturePad: Unobtrusive Wearable Interaction Devices. In Proceedings of the 5th IEEE International Symposium on Wearable Computers (ISWC '01). IEEE.

9. Rico, J., and Brewster, S.: Usable gestures for mobile interfaces: evaluating social acceptability. In Proceedings of the SIGCHI Conference on Human Factors in Computing Systems (CHI '10). pp. 887-896. ACM.

10. Tung, Y., Hsu, C., Wang, H., Chyou, S., Lin, J., Wu, P., Valstar, A., and Chen, M.: UserDefined Game Input for Smart Glasses in Public Space. In Proceedings of the 33rd Annual ACM Conference on Human Factors in Computing Systems (CHI '15). pp. 3327-3336. ACM. 